\title{
Ultravilolet-radiation-induced graft polymerization of acrylamide onto the melt-blown polypropylene filter element by dynamic method
}

\author{
WU Lin-lin ${ }^{1}$, WU Guang-xia ${ }^{1, *}$, XU Shu-guang ${ }^{1}$, ZHONG Hui $^{1}$, SHEN Ying-jie ${ }^{1}$, LIU He-zhi $^{2}$, \\ HUANG Zheng-ming ${ }^{3}$, YANG Xin-bo ${ }^{4}$, YUAN Zong-huan ${ }^{5}$, TANG Lian-yi ${ }^{5}$, \\ LIN Xiang-wei ${ }^{5}$, ZHANG Shao-lai $^{6}$, ZHANG Wei-jun $^{6}$ \\ 1. Research Center for Eco-environmental Sciences, Chinese Academy of Sciences, Beijing 100085, China. E-mail: lindawwu@sina.com \\ 2. Insititute for Drug and Instrument Control of Health Dept GLD of PLA, Beijing 100071, China \\ 3. 302 Hospital of PLA, Beijing 100039, China \\ 4. 301 Hospital of PLA, Beijing 100853, China \\ 5. The Greatwall Pharmaceutical Factory of Beijing, Beijing 100071, China \\ 6. Beijing Tong Ren Tang Company Limited Scientific Research Institute, Beijing 100011, China
}

Received 20 December 2006; revised 21 June 2007; accepted 1 July 2007

\begin{abstract}
By dynamic method under UV irradiation, commercial melt-blown polypropylene (PPMB) filter element was modified with acrylamide (AAm) using benzophenone (BP) as initiator. Attenuated total reflection-Fourier transform infrared spectroscopy and scanning electron microscope verified that polyacrylamide chain was grafted on the fiber surface of PPMB filter element. Elemental content analysis with energy dispersive X-ray of fibers revealed that the polymerization content in the inner part of filter element was relatively higher than that in the outer. Degree of grafting changed with initiator concentration, monomer concentration, reaction temperature and reached $2.6 \%$ at the reaction condition: $C_{\mathrm{BP}}=0.06 \mathrm{~mol} / \mathrm{L}, C_{\mathrm{AAm}}=2.0 \mathrm{~mol} / \mathrm{L}$, irradiation time: 80 min, temperature: $60^{\circ} \mathrm{C}$. Relative water flux altered with the hydrophilicity and pore size of filter element. In the antifouling test, the modified filter gave greater flux recovery (approximately 70\%) after filtration of the water extract of Liuweidihuang, suggesting that the fouling layer was more easily reversible due to the hydrophilic nature of the modified filter.
\end{abstract}

Key words: melt-blown polypropylene filter element; UV-induced graft polymerization; surface modification; dynamic method

\section{Introduction}

Melt-blown polypropylene (PPMB) filter element is made of polypropylene fibers twisted according to different density. It is a new type of microporous filter which has been widely used in the field of water treatment, food, electronic, pharmaceutical and chemical industry, because it has outstanding filtration capability, such as reliable filtration precision, wide chemical compatibility, high fluxes, long filtration period and low cost $(\mathrm{Li}$ and Ye, 2001). However, as many synthetic polymer used as commercial materials, PPMB filter element is hydrophobic due to the low surface energy of polypropylene. In some extent, this drawback limits its potential application in oilwater separation and some pharmaceutical areas.

As we know, there is a significant interest among both academic and industrial scientific communities in developing methods for modifying the polymer materials surface. To enhance wettability, printability, adhension

Project supported by the Natural Science Foundation of Beijing (No. 2051002) and the Science and Technology Programme of Beijing (No. D0205004040421). *Corresponding author. E-mail: wgxzhlj@yahoo.com.cn. and compatibility with other materials or with biological components without altering the bulk properties, different surface functional groups were grafted on substrate surface by irradiation with various sources (UV, gamma ray, electron beam, etc.) (Ma et al., 2001). Among these methods, due to its advantages of low cost operation and mild reaction conditions, UV has been extensively applied for surface graft polymerization with the aid of a photoinitiator or photosensitizer, such as benzophenone (BP) (Shanmugharaj et al., 2006).

In our previous research, in order to adjust the diameter of membrane pores, gas-initiation under UV and liquidgrafting polymerization on the surface of polysulfone hollow-fiber ultrafiltration membrane has been studied by dynamic method (Shen et al., 2005). Compared with traditional static method and two-step process, dynamic method can make the initiator which is irradiated under UV transmitted to the place difficult to be irradiated directly. After the initiator was circulated by a peristaltic pump for a certain period, the solution of monomer is pumped through the polymer to complete graft polymerization.

As a widely used polymer material, the hydrophilic 
modification of polypropylene (PP) has been extensively studied. In these studies, various forms of PP, such as film (Chen et al., 1999), fiber (Huang et al., 2006) and membrane (Yu et al., 2005), were used. However, there are few studies on the hydrophilic modification of PP material with special figuration, for example filter element. In this report, PPMB filter element was grafted with acrylamide by dynamic method to alter its hydrophobic property. Attenuated total reflection-Fourier transform infrared spectroscopy and scanning electron microscope (SEM) and elemental content analysis with energy dispersive X-ray (EDX) were used to characterize the hydrophilic modification of PPMB filter element. Several graft parameters (initiator concentration, monomer concentration, irradiation time, graft temperature) were examined to understand their influences on the degree of grafting and water flux. Furthermore, the antifouling capability of modified filter was evaluated by the filtration of water extracts of Liuweidihuang.

\section{Experimental}

\subsection{Materials}

PPMB filter element (absolute rating $=1.0 \mu \mathrm{m}$; outer diameter $=6.4 \mathrm{~cm}$; inner diameter $=2.8 \mathrm{~cm}$ ) was procured from Beijing Liquid Filter New Material Tech. Co., Ltd. To save solvents and calculate the degree of grafting accurately, filter element was cut into segments of $1.0 \mathrm{~cm}$ in length (Fig.1). Their average weight was 5.3931 \pm 0.0661 g. Before grafting, filter was flushed with deionized water and ethanol to remove the residual solvents in production. Then it was dried to constant weight in a vacuum drier $\left(60^{\circ} \mathrm{C}\right)$ and weighed with an analytical balance, which had an accuracy of $0.1 \mathrm{mg}$.

BP, acrylamide (AAm) and ethanol were obtained from Peking Chemistry and Reagent Co. Nitrogen was of medium purity.

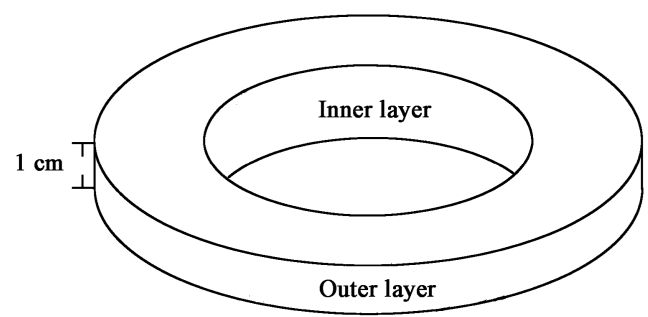

Fig. 1 Schematic of filter element in this experiment.

\subsection{Dynamic photografting procedures}

A schematic representation of the process is shown in Fig.2. This photochemical reactor system is devised and made in our laboratory of Chinese Academy and Sciences. It is equipped with a $1000-\mathrm{W}$ high pressure mercury lamp and a quartz glass which permits UV light to penetrate.

The procedures for dynamic photografting by liquidinitiation under UV and liquid-polymerization are described as follows. In the first step, BP solution bubbling with $\mathrm{N}_{2}$ was heated in a water bath. After exposed to UV irradiation under nitrogen atmosphere, BP was excited by

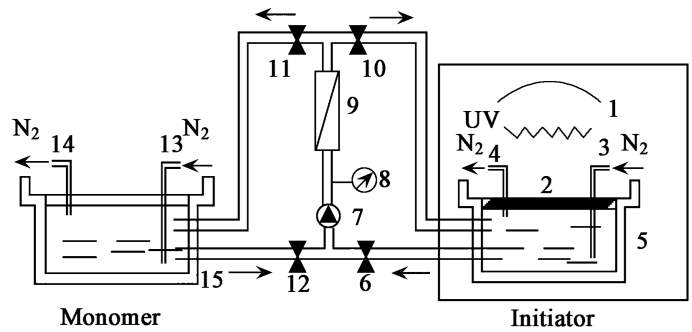

Fig. 2 Schematic of photografting process by dynamic method. (1) UV lamp; (2) quartz cover; $(3,13) \mathrm{N}_{2}$ inlet; $(4,14) \mathrm{N}_{2}$ outlet; (5) initiator solution; $(6,10,11,12)$ valve; (7) flowmeter; (8) manometer; (9) filter.

absorption in near UV (340-360 nm) to a singlet state which was short-lived and transformed to a triplet state. The triplet states could abstract hydrogen from solvent to generate radicals and benzpinacol radicals. In the second step, at first it was essential to utilize nitrogen to purge all the pipe lines lest oxygen would terminate the forming of free radicals during irradiating phase. Then photoinduced initiator was transmitted through the filter element, the triplet states and semipinacol radicals abstracted hydrogen from the polypropylene fiber to generate surface radicals. The surface radicals and semipinacol radicals combined to form dormant species. These dormant species could be opened under a certain temperature. In the third step, acrylamide dissolved in the proper solvent was pumped through the filter element to complete polymerization.

\subsection{Characterization}

Fourier transform infrared spectroscopy (FTIR) spectra was recorded on a Nicolet Avatar 360 FTIR Spectrometer (Thermo Nicolet Corporation) equipped with an ATR crystal accessory. For each measurement, 32 scans were performed at a resolution of $\pm 4.0 \mathrm{~cm}^{-1}$. Samples were prepared for FTIR by placing them in a vacuum drier overnight at $50^{\circ} \mathrm{C}$.

The morphology of the virgin and modified fiber samples was analyzed with SEM (JSM-6301F). Samples were dried by Critical Point Dryer and then sputtered with gold. All SEM analysis was performed under $15 \mathrm{keV}$ at $20^{\circ} \mathrm{C}$.

EDX (GENESIS, EDAX) spectra of samples in different locations of the modified filter element were performed with the same area observed with SEM. According to the spectra, elemental compositions $(\mathrm{C}, \mathrm{N}, \mathrm{O})$ were calculated and the PP-g-AAm distribution of the modified filter element could be analyzed.

Degree of grafting (DG) was established gravimetrically. After grafting, the modified filter element was flushed with ethanol firstly to remove the residual monomer and initiator. Then, it was washed with hot deionized water (approximately $80^{\circ} \mathrm{C}$ ) for at least $24 \mathrm{~h}$ to remove the soluble homopolymer and dried in a vacuum drier $\left(60^{\circ} \mathrm{C}\right)$ until constant weight. Finally, weight gain measurements were calculated applying the following equation:

$\mathrm{DG}=\frac{W_{1}-W_{0}}{W_{0}} \times 100 \%$

where, $W_{0}$ denotes the weight of the virgin filter element, and $W_{1}$ presents the weight of the filter element after 
grafting, obtained by an analytic balance with an accuracy of $0.1 \mathrm{mg}$.

Relative water flux testing was used to demonstrate the effects of DG on water flux of the grafted filter element. Filter elements were filtered with pure water at $0.01 \mathrm{MPa}$ for at least $10 \mathrm{~min}$, then water fluxes were measured by timed collection until the stable values were obtained.

Relative water flux was calculated as follows:

$F_{\mathrm{r}}=F_{\mathrm{g}} / F_{0}$

where, $F_{\mathrm{g}}$ is the water flux of grafted filter element; $F_{0}$ is the water flux of virgin filter element. If $F_{\mathrm{r}}=1$, it means that water flux was not altered; if $F_{\mathrm{r}}>1$ or $F_{\mathrm{r}}<1$, it means that water flux was affected by different grafting efficiencies.

Filtration test: In this test, traditional Chinese medicine compound-Liuweidihuang was used to check the antifouling characteristic of virgin and modified filter element. The water extract of Liuweidihuang was circulated through the filter by a peristaltic pump at $0.02 \mathrm{MPa}$. The permeate flux was measured using a measuring cylinder and then the permeate solution was returned to the feed bank to keep the total system volume constant. When the filtration process

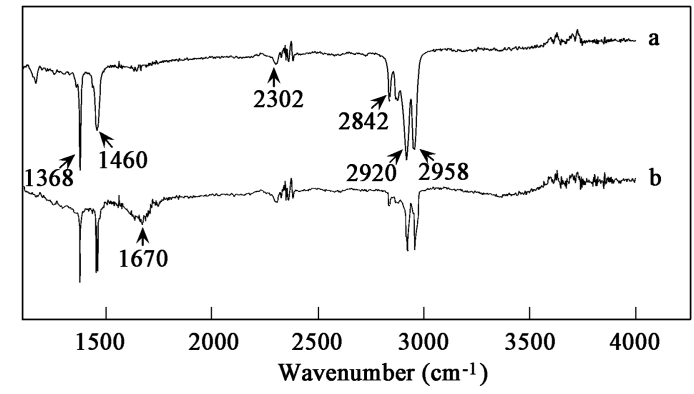

Fig. 3 FTIR spectra of the fiber surface. (a) virgin fibers; (b) modified fibers. Condition: $C_{\mathrm{BP}}=0.06 \mathrm{~mol} / \mathrm{L} ; C_{\mathrm{AAm}}=2.0 \mathrm{~mol} / \mathrm{L}$; irradiation time: $80 \mathrm{~min}$; temp. $=60^{\circ} \mathrm{C}$. was finished, the filter element was back-washed by pure water. Before filtration and after washing, the pure water flux was measured to check the antifouling nature of the filter element.

\section{Results and discussion}

\subsection{FTIR spectra analysis}

Figure 3 shows the FTIR spectra of virgin and modified fiber of PPMB filter element. The modified sample was taken from the inner layer. Both in the virgin and modified fiber, the peaks at 2920,2842 and $2958 \mathrm{~cm}^{-1}$ corresponded to $-\mathrm{C}-\mathrm{H}$ asymmetric and symmetric stretching of $-\mathrm{CH}_{3}$ and $-\mathrm{CH}_{2}$ groups. The peaks at $1368 \mathrm{~cm}^{-1}$ and $1460 \mathrm{~cm}^{-1}$ were due to $-\mathrm{C}-\mathrm{H}$ stretching of $-\mathrm{CH}_{3}$ group. The FTIR spectra of the modified fiber surface showed a new and broad peak at $1670 \mathrm{~cm}^{-1}$, which could be assigned to the combined motion of $-\mathrm{NH}_{2}$ and $\mathrm{C}=\mathrm{O}$ stretching in the $-\mathrm{CONH}_{2}$ group. This new peak confirmed the presence of polyacrylamide on the surface of modified fiber. As shown in the spectra, there was a small peak at about $2302 \mathrm{~cm}^{-1}$ both in the virgin and modified samples. However, this peak was absent in the FTIR spectra of pure polypropylene. It was speculated that the presence of this peak was due to the processing technic of PPMB filter element.

\subsection{Scanning electron micrographs and EDX analysis}

Figure 4 shows the SEM microphotographs of virgin and modified fiber surface. SEM microphotographs revealed that virgin fibers were smooth and homogeneous (Fig.4a). When the filter element was subjected to acrylamide modification for $10 \mathrm{~min}$ irradiation of $\mathrm{BP}\left(C_{\mathrm{BP}}=0.06 \mathrm{~mol} / \mathrm{L}\right.$, $\left.C_{\mathrm{AAm}}=0.5 \mathrm{~mol} / \mathrm{L}\right)$, the fiber surface changed slightly compared with the virgin fibers (Fig.4b). However, under the enhanced reaction condition $\left(C_{\mathrm{BP}}=0.06 \mathrm{~mol} / \mathrm{L}, C_{\mathrm{AAm}}\right.$ $=0.5 \mathrm{~mol} / \mathrm{L}$, irradiation time: $80 \mathrm{~min}$ ), it was obvious

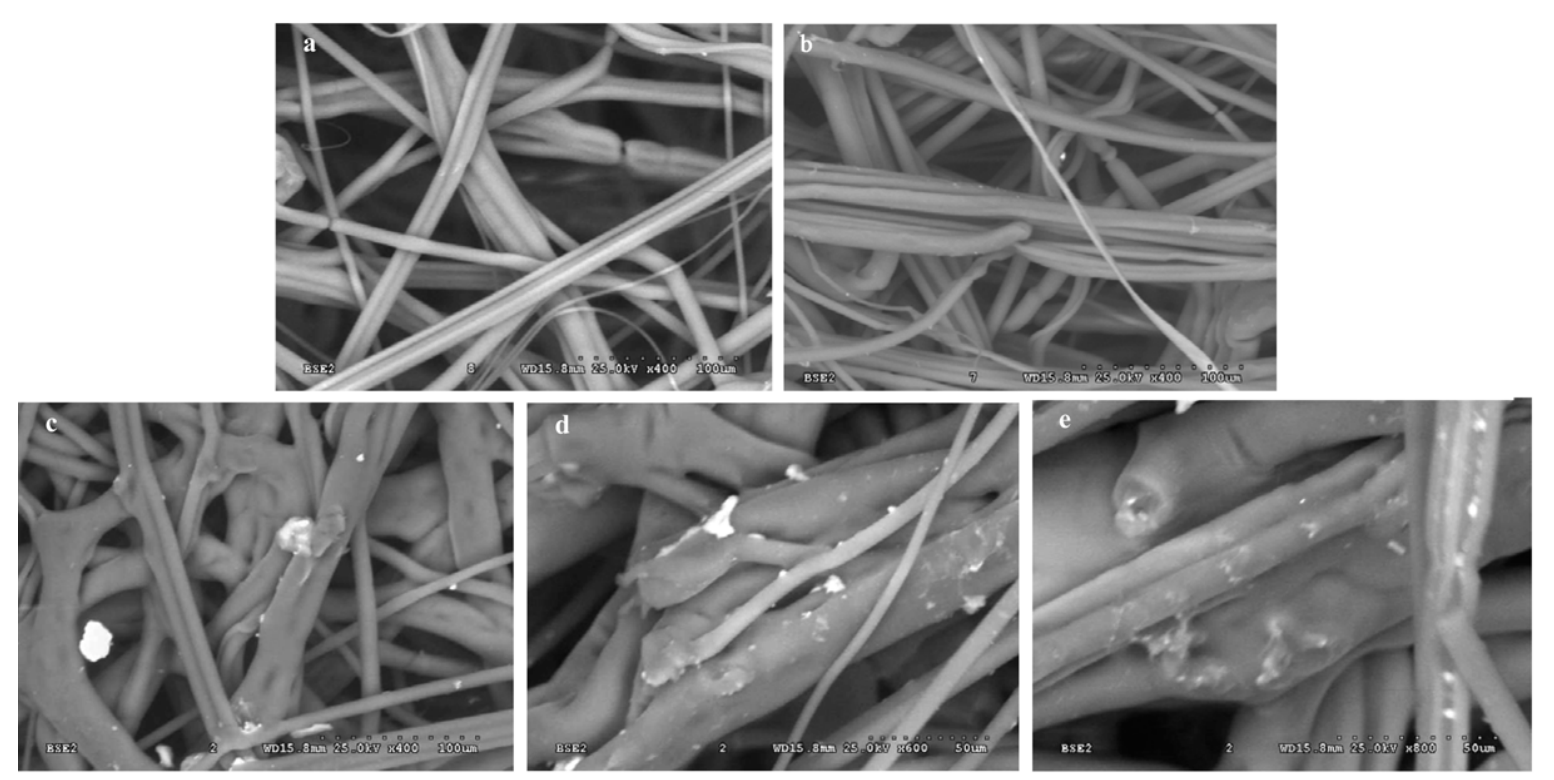

Fig. 4 Scanning electron micrographs of fibers. (a) 400 times surface of virgin fibers and (b) 400 times surface of modified fibers under the reaction condition: $C_{\mathrm{BP}}=0.06 \mathrm{~mol} / \mathrm{L}, C_{\mathrm{AAm}}=0.5 \mathrm{~mol} / \mathrm{L}$ and irradiation time $10 \mathrm{~min}$, (c) 400, (d) 600, (e) 800 times surface of modified fibers under the reaction condition: $C_{\mathrm{BP}}=0.06 \mathrm{~mol} / \mathrm{L}, C_{\mathrm{AAm}}=0.5 \mathrm{~mol} / \mathrm{L}$, irradiation time: $80 \mathrm{~min}$. 
that the fibrous PP matrix was grafted with the villiform polymer (Fig.4e). It revealed that at a short irradiation time there could not form enough free radicals on the fiber surface, therefore the chances of polymerization were less.

In the graft process, both the initiator and monomer solution transmitted the filter from the inner layer to the outer. Thus, it was speculated that such a flow mode would affect the grafted polymer distribution in the filter. To understand the polymerization distribution, elemental compositions depending on the different locations of the filter were analyzed with EDX. The four samples were taken from the inner layer to the outer with the distances of $0,0.18,0.9$, and $1.6 \mathrm{~cm}$ (location relative to the whole thickness is $0 \%, 10 \%, 50 \%$ and $90 \%$ ). For each sample, the characteristic peaks of $\mathrm{C}, \mathrm{N}, \mathrm{O}$ were scanned and the atom contents were calculated from the scanning plot with the same magnification. As shown in Fig.5, the content of $\mathrm{N}, \mathrm{O}$ decreased slightly with the increased distance. This illustrated that the polymerization content in the inner layer was higher than that in the outer. This phenomenon could be explained that: on one hand, when the initiator solution flowed from the inner layer to the outer, the free radicals would be consumed firstly by the inner matrix and this would reduce the amount of free radicals which arrived at the outer layer; on the other, due to the separation of initiation and polymerization phase, graft polymerization firstly took place on the inner layer of filter element, and the grafted polymer chains would hinder the polymerization in the outer layer in some extent.

\subsection{Effect of irradiation time on the degree of grafting and relative water flux}

The influence of the irradiation time on the DG is shown in Fig.6. The DG was about $2.0 \%$ with the irradiation time up to $80 \mathrm{~min}$. At first, as the reaction time increased, the number of surface radicals and dormant species on the fiber backbone increased. As a result, the extent of initiation and propagation of photografting copolymerization also increased with the irradiation time. On the irradiation time of $80 \mathrm{~min}$, the formation of surface radicals and dormant species was in equilibrium and would not increase with irradiation time. Thus, the DG leveled off.

With the DG increasing, at first the relative water flux increased to 1.15 , but decreased to be less than 1.0 after

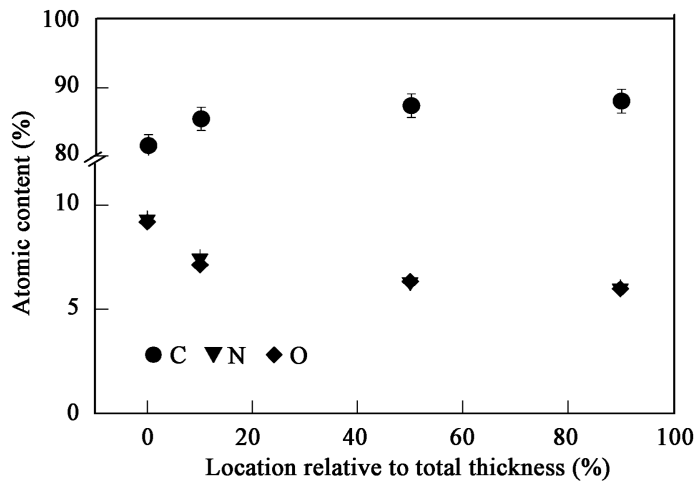

Fig. 5 Compositions from EDX analyses depending on sampling locations from the inner layer to the outer.

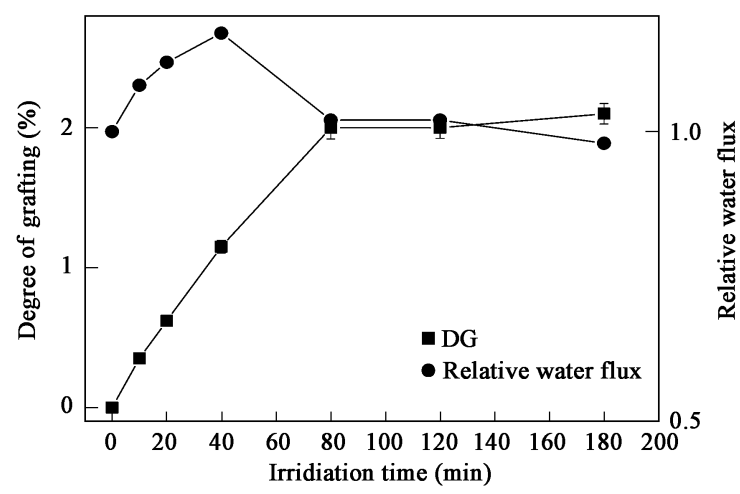

Fig. 6 Influence of the irradiation time on DG and relative water flux. Reaction conditions: $C_{\mathrm{BP}}=0.06 \mathrm{~mol} / \mathrm{L} ; C_{\mathrm{AAm}}=0.5 \mathrm{~mol} / \mathrm{L}$; temp. $=$ $60^{\circ} \mathrm{C}$.

the DG continued to increase. This phenomenon stated that the hydro-polymer chain of acrylamide was attached on the fibers. Furthermore, the balance between hydrophilicity increase and photocrosslink led to the change of relative water flux. Since the inner layer of filter element had the smaller pore sizes compared with other layers, the polymer formation on the inner fibers would inevitably affect the water flux.

\subsection{Effect of monomer concentration and initiator con- centration on the degree of grafting and relative water flux}

In the dynamic method, initiator solution and monomer solution were re-circled back, respectively. As in the static method, both the initiator concentration and the monomer concentration would affect the DG.

During the initiation phase, free radicals formed by UV irradiation must be quickly pumped to the fibers surface of filter element to keep their activity. The amount of grafted polymer on the filter element at different initiator concentrations was shown in Fig.7. There was a maximum DG was observed with the increasing BP concentration $(0$ $0.24 \mathrm{~mol} / \mathrm{L})$. As we know, the direct effect of higher BP concentration is to increase reaction rate, but the indirect effect of higher BP concentration is to increase light absorption, lowering the UV intensity at the interface of the BP solution (Ma et al., 2001). On the other hand, higher

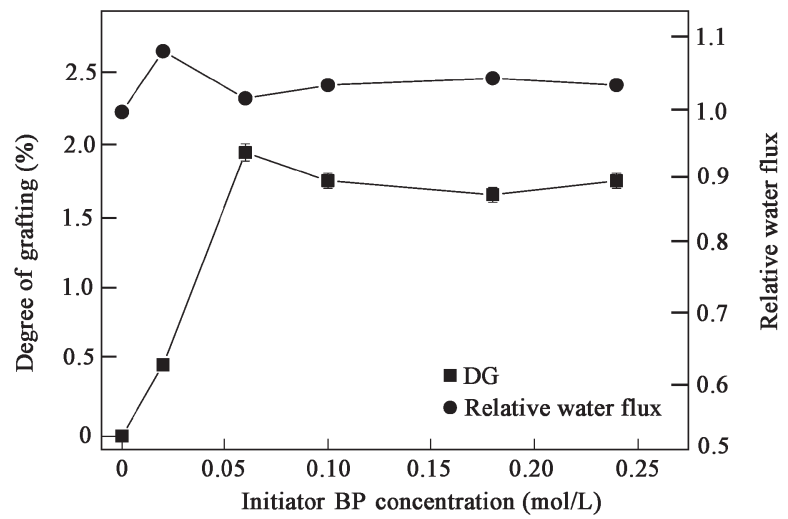

Fig. 7 Influence of initiator BP concentration on DG and relative water flux. Reaction conditions: $C_{\mathrm{AAm}}=0.5 \mathrm{~mol} / \mathrm{L}$; irradiation time: $80 \mathrm{~min}$; temp. $=60^{\circ} \mathrm{C}$ 
initiator concentration may result in more terminations of the initiator free radicals. These may lead to the decrease of DG.

The effects of different monomer concentrations were also examined. As shown in Fig.8, DG increased steadily from 0 to $2.4 \%$ when monomer concentration was up to 1.0 $\mathrm{mol} / \mathrm{L}$, and beyond this concentration, DG started to level off from $2.6 \%$ at $2.0 \mathrm{~mol} / \mathrm{L}$ the concentration to $2.3 \%$ at $3.0 \mathrm{~mol} / \mathrm{L}$. This result can be explained as follows: Before the DG reached $2.6 \%$, the extent of grafting increased with the increasing amount of monomer; however, when the monomer solution kept on increasing, the homopolymerization of acrylamide also enhanced. This may cause the hindrance in the grafting polymerization, particularly through the coupling of growing polymer chains. Thus, the DG decreased.

\subsection{Effect of temperature on the degree of grafting}

The graft copolymerization of AAm onto PPMB filter element was carried out at different temperatures ranging from 40 to $80^{\circ} \mathrm{C}$, respectively (Fig.9). At the same monomer concentration, the DG increased with the reaction temperature from 40 to $70^{\circ} \mathrm{C}$ but did not change dramatically with a further increase. This may be attributed to the formation of the more homopolymer beyond $70^{\circ} \mathrm{C}$. In addition, at higher temperatures, various hydrogen abstraction and chain-transfer reactions might have been

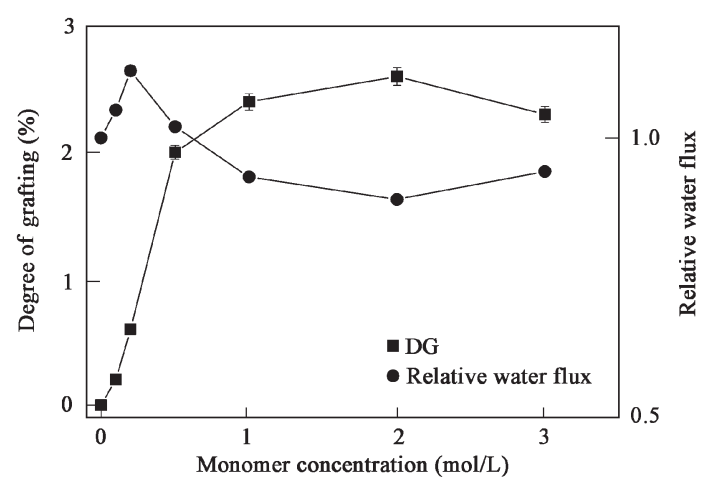

Fig. 8 Influence of the monomer concentration on DG and relative water flux. Reaction conditions: $C_{\mathrm{BP}}=0.06 \mathrm{~mol} / \mathrm{L}$; irradiation time: $80 \mathrm{~min}$; temp. $=60^{\circ} \mathrm{C}$.

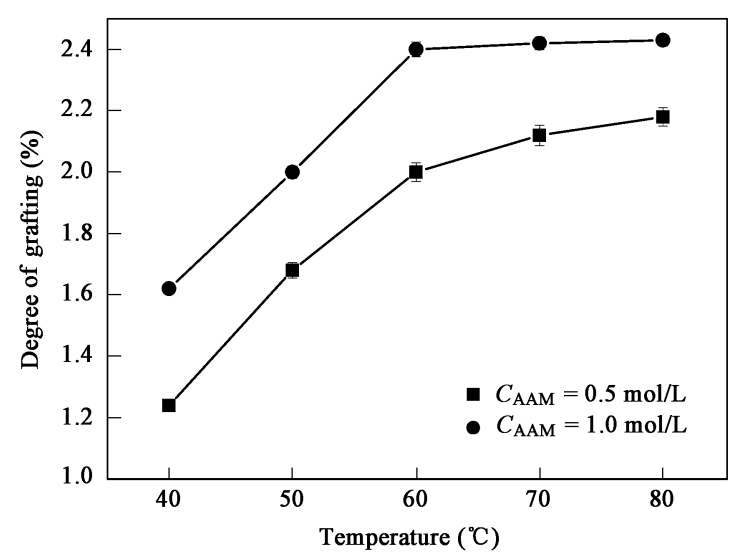

Fig. 9 Influence of the temperature on DG. Reaction conditions: $C_{\mathrm{BP}}=$ $0.06 \mathrm{~mol} / \mathrm{L}$; irradiation time: $80 \mathrm{~min}$. accelerated and led to the decrease in the DG (Thaker and Trivedi, 2005).

\subsection{Effect of different solvents on grafting polymeriza- tion reaction}

As we know, different solvents have different effects on the formation of free radicals. This effect is largely related to the liability of hydrogen atoms in the solvent and wettability of substrate by the solvent. It had been reported (Yang and Ranby, 1996) that the photoreduction reactivity for different types of hydrogen typically follows tertiary $\mathrm{H}>$ secondary $\mathrm{H}>$ primary $\mathrm{H}>\mathrm{H}$ in phenol ring. Here, both ethanol and acetone were used as initiator solvents. Fig.10 shows the DG in the two solvents. Compared with ethanol, acetone was a better solvent of BP and PP fibers. This was beneficial for initiator to diffuse on the surface of fibers. Although the number of semipinacol radicals in the acetone may be effected by the higher hydrogen bond strength $\left(\mathrm{CH}_{3} \mathrm{OCH}_{2}-\mathrm{H} 93.0 \pm 1.0\right.$ $\mathrm{kcal} / \mathrm{mol}, \mathrm{CH}_{3} \mathrm{COCH}_{2}-\mathrm{H} 98.3 \pm 1.8 \mathrm{kcal} / \mathrm{mol}$, data from the Handbook of Chemistry and Physics), acetone still showed a higher degree of grafting.

As for choosing the solvent of monomer, firstly the solvent should not terminate the free radicals. Another crucial factor which should be considered was that it must be a good solvent for both monomer and initiator. When the initiation step was finished, a small part of initiator solvent was left in the pipeline. Thus, the graft polymerization reaction would be seriously diminished when the monomer solution was pumped through the pipelines, if the solvent of monomer could not dissolve the initiator.

\subsection{Filtration test}

As we know, there exists a large amount of macromolecular substances (e.g. proteins, balata) in the water extracts of traditional Chinese medicine. When the filter element was used in the filtration of water extracts, the antifouling capability was very important in keeping the constant filtration besides other operation parameters. In this experiment, the water extracts of Liuweidihuang was used to examine the antifouling characteristic of virgin and modified filter element.

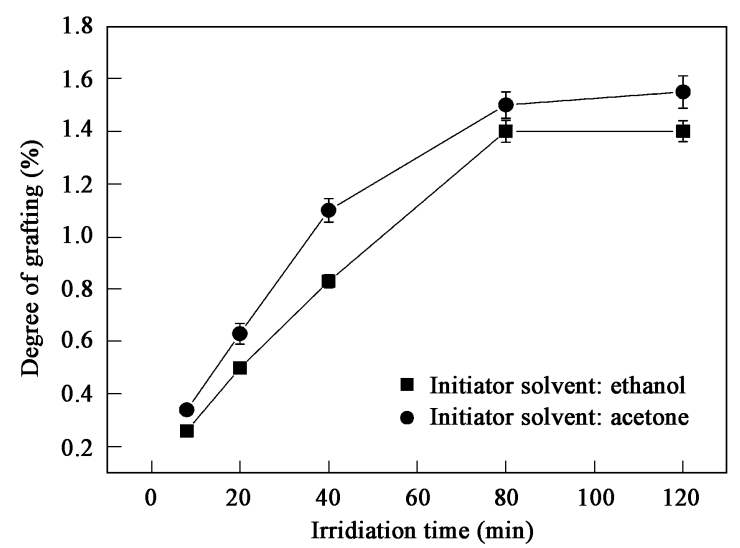

Fig. 10 Influence of different initiator solvents on DG. Reaction conditions: $C_{\mathrm{BP}}=0.06 \mathrm{~mol} / \mathrm{L} ; C_{\mathrm{AAm}}=0.5 \mathrm{~mol} / \mathrm{L}$; irradiation time: $80 \mathrm{~min}$; temp. $=60^{\circ} \mathrm{C}$; both monomer solvents are ethanol. 


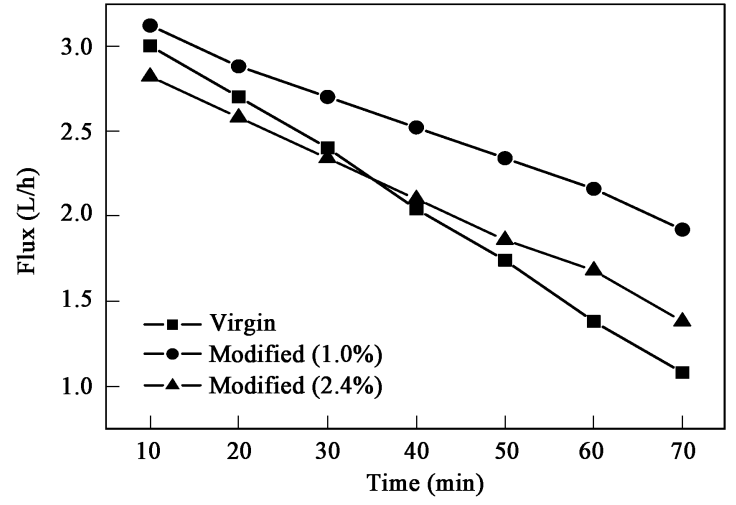

Fig. 11 Fluxes for virgin and grafted filter during the filtration of water extracts of Liuweidihuang.

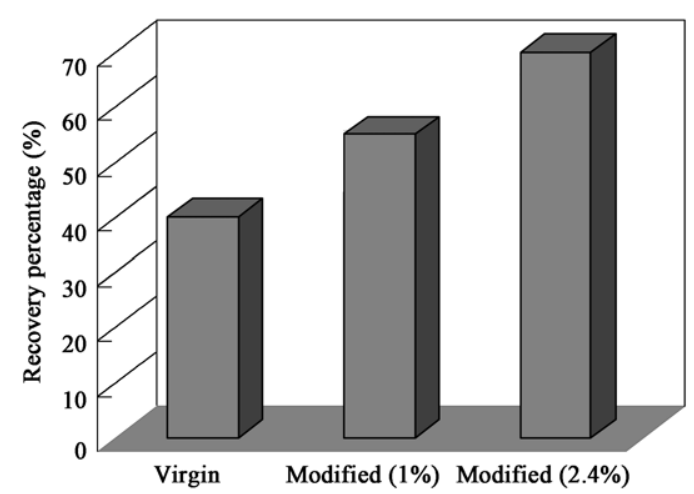

Fig. 12 Flux recovery efficiencies with water flushing at the end of filtration.

As shown in Fig.11, the fluxes of both the virgin and grafted filter element declined with the operation time due to the adsorption and accumulation of macromolecular substances. However, the virgin filter element showed a more dramatic decrease of flux than the grafted. This illustrated that during filtration, the modified filter element had more effective fouling-resistance because of the enhanced hydrophilicity. Fig.11 also showed that the fluxes of both the virgin and grafted filter were very low. This phenomenon was as a result of the filtration under a constant pressure (0.02 MPa). Actually, in the industrial application, the operation pressure should be changed continuously to diminish the flux loss ( $\mathrm{Li}$ and Ye, 2001).

After the filter was back-washed with the pure water, the flux recovery efficiency is shown in Fig.12. The flux recovery of the grafted filter element (DG was 2.4\%) reached $70 \%$. However, the flux recovery of the virgin was only $40 \%$. This suggested that although the fouling existed in both the virgin and grafted filter element, it could be reversed more easily for the grafted filter element by water flushing. This could be contributed to the more hydrophilic nature of modified filter.

\section{Conclusions}

In this paper, PPMB filter element was segmented and altered its hydrophilicity by dynamic method. This method could realize the grafting polymerization with loss of UV irradiation. The chemistry and topography of the modified filter were verified with FTIR, SEM and EDX. Also, some factors in the grafting reaction were studied to find the optimum reaction conditions. Furthermore, the filtration of the water extracts of Liuweidihuang confirmed that the modified filter element had more effective foulingresistance due to the enhanced hydrophilicity. Based on these results, the different modification of materials with some special figurations can be realized by dynamic method according to changing various monomers.

\section{References}

Chen J, Nho C Y, Kwon O H et al., 1999. Grafting copolymerization of acrylamides onto preirradiated PP films[J]. Radiation Physics and Chemistry, 55: 87-92.

Huang F L, Wei Q F, Wang X Q et al., 2006. Dynamic contact angles and morphology of PP fibers treated with plasma[J]. Polymer Testing, 25: 22-27.

Li Y, Ye S Y, 2001. The characteristics and operating condition of melt-brown polyproylene fiber sieve-core[J]. Filtration and Separation, 11: 38-40.

Ma H, Davis R H, Bowman C N, 2001. Principal factors affecting sequential photoinduced graft polymerization[J]. Polymer, 42: 8333-8338.

Shanmugharaj A M, Kim J K, Ryu S H, 2006. Modification of rubber surface by UV surface grafting[J]. Applied Surface Science, 252(16): 5714-5722.

Shen Y J, Wu G X, Xu S G, 2005. Gas-initiation under UV and liquid-grafting polymerization on the surface of polysulfone hollow fiber ultrafiltration membrane by dynamic method[J]. Journal of Environmental Sciences, 17: 465468.

Thaker M D, Trivedi H C, 2005. Ultraviolet-radiation-induced graft copolymerization of methyl acrylate onto the sodium salt of partially carboxymethylated guar gum[J]. Journal of Applied Polymer Science, 97: 1977-1986.

Yang W T, Ranby B, 1996. Bulk surface photografting process and its application: I. Reaction and kinetics[J]. Journal of Membrane Sciences, 62: 533-543.

Yu H Y, Xu Z K, Xie Y J et al., 2005. Flux enhancement for polypropylene microporous membrane in a SMBR by the immobilization of poly(N-viny1-2-pyrrolidone) on the membrane surface[J]. Journal of Membrane Science, 279(1/2): 148-155. 